\title{
The FFAG return loop for the CBETA Energy Recovery Linac
}

\author{
J.S. Berg
}

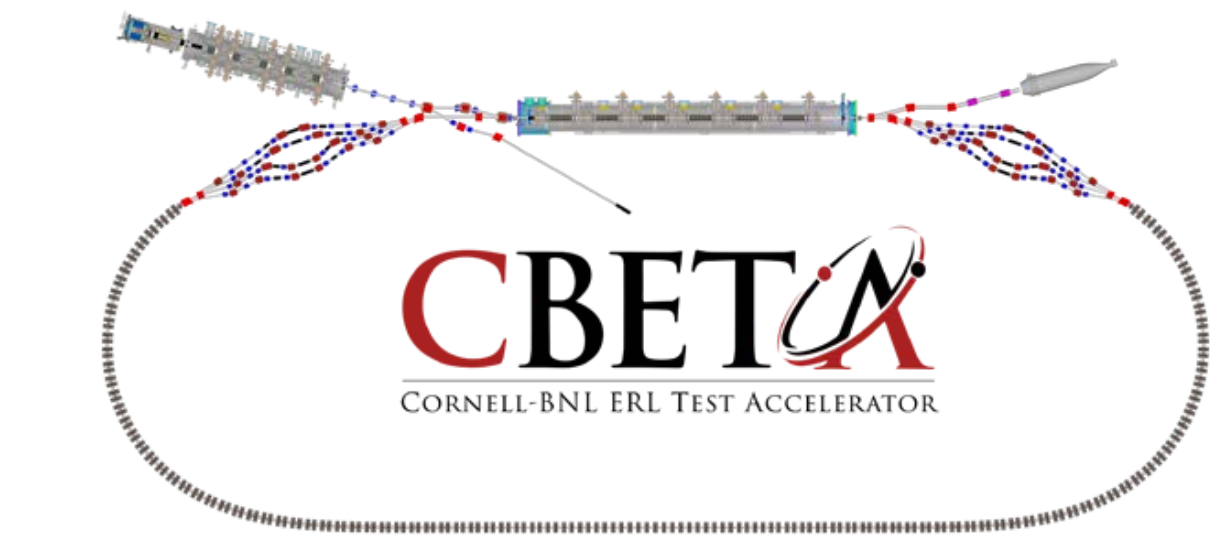

Collider-Accelerator Department, Brookhaven National Laboratory, Upton NY 11973 U.S. Department of Energy

Office of Science, Office of Nuclear Physics

Cornell Laboratory for Accelerator-Based Sciences and Education, Ithaca, NY 14850

Funded by NYSERDA contract 102192

Notice: This document has been authorized by employees of Brookhaven Science Associates, LLC under Contract No. DE-SC0012704 with the U.S. Department of Energy. The United States Government retains a non-exclusive, paid-up, irrevocable, world-wide license to publish or reproduce the published form of this document, or allow others to do so, for United States Government purposes. 


\section{DISCLAIMER}

This report was prepared as an account of work sponsored by an agency of the United States Government. Neither the United States Government nor any agency thereof, nor any of their employees, nor any of their contractors, subcontractors, or their employees, makes any warranty, express or implied, or assumes any legal liability or responsibility for the accuracy, completeness, or any third party's use or the results of such use of any information, apparatus, product, or process disclosed, or represents that its use would not infringe privately owned rights. Reference herein to any specific commercial product, process, or service by trade name, trademark, manufacturer, or otherwise, does not necessarily constitute or imply its endorsement, recommendation, or favoring by the United States Government or any agency thereof or its contractors or subcontractors. The views and opinions of authors expressed herein do not necessarily state or reflect those of the United States Government or any agency thereof. 


\title{
The FFAG Return Loop for the CBETA Energy Recovery Linac
}

\author{
J. Scott Berg ${ }^{1}$ \\ Brookhaven National Laboratory; P. O. Box 5000; Upton, NY, 11973-5000; USA
}

\begin{abstract}
The CBETA energy recovery linac uses a single fixed field alternating gradient (FFAG) beam line to return the beam for electron beams with four energies, ranging from $42 \mathrm{MeV}$ to $150 \mathrm{MeV}$. To keep the beam line compact, the ends of the return line have a small radius of curvature, but the central part of the return line is straight. These are connected by transition lines that adiabatically change from one to the other. We first describe the design or the arc cell. We then describe how a straight cell is created to be a good match to this arc cell. We then describe the design of the transition line between them. The design process makes use of field maps for the desired magnets. Because we switch magnet types as we move from the arc, through the transition, and into the straight, there are discrete jumps in the fields that degrade the adiabaticity of the transition, and we describe corrections to manage that.
\end{abstract}

\section{The Basic Parameters}

CBETA is an energy recovery linac that will make 4 accelerating passes through the linac, and return the beam to the linac using a single fixed field alternating gradient (FFAG) return line. At the ends of the linac are 4 spreader/combiner lines, each of which transports a single energy from the linac to the FFAG line or from the FFAG line back to the linac.

The FFAG return line has arcs at it ends with a relatively small bending radius to keep the machine compact. Completing the return to the linac requires a section that is straight or with larger radius of curvature to connect the two arcs. We use an adiabatic transition section connecting each arc to a straight between them.

Table 1 describes the basic requirements for the FFAG line design. The energies correspond to a four-pass energy recovery linac with a $6 \mathrm{MeV}$ injection energy. The minimum drift lenghts result from allowing space for various devices (the short drift allows for a button beam position monitor (BPM), the long drift will allow for a wide variety of devices) and any overhang of magnet hardware. The radius of curvature is a result of a space limitation. The magnet lengths and maximum energy are parameters related to an earlier design using an iron-dominated magnet design, but are reasonable choices that were kept for efficiency in the design process.

Each arc has 16 cells, giving 80 degrees of bend. The transition will be designed with a symmetry such that the

\footnotetext{
Email address: jsberg@bnl.gov (J. Scott Berg)

${ }^{1}$ This manuscript has been authored by employees of Brookhaven Science Associates, LLC under Contract No. DE-SC0012704 with the U.S. Department of Energy. The United States Government retains a non-exclusive, paid-up, irrevocable, world-wide license to publish or reproduce the published form of this manuscript, or allow others to do so, for United States Government purposes.
}

Table 1: Basic parameters for the FFAG return line.

\begin{tabular}{lr}
\hline Total energy, pass 1 (MeV) & 42 \\
Total energy, pass $2(\mathrm{MeV})$ & 78 \\
Total energy, pass 3 (MeV) & 114 \\
Total energy, pass $4(\mathrm{MeV})$ & 150 \\
Focusing quadrupole length (mm) & 133 \\
Defocusing magnet length $(\mathrm{mm})$ & 122 \\
Minimum short drift length (mm) & 66 \\
Minimum long drift length (mm) & 123 \\
Arc radius of curvature, approximate $(\mathrm{m})$ & 5.1 \\
Arc cell bend angle (deg.) & 5 \\
Cells per arc & 16 \\
Cells per transition section & 24 \\
\hline
\end{tabular}

average bend per cell is half the arc cell bend angle. Thus each transition section supplies 60 degrees of bend. Thus each spreader/combiner supplies the remaining 40 degrees of bend for half the machine.

Every focusing quadrupole will have a horizontal corrector (vertical dipole field), while every defocusing magnet will have a vertical corrector.

\section{Arc Cell}

The arc cell is the basic building block for the FFAG beam line. An illustration is given in Fig. 1. The basic cell is a doublet, consisting of a focusing quadrupole and a combined function magnet with a dipole and defocusing quadrupole component. The geometry is defined to relate to the vacuum chamber design, which consists of $42 \mathrm{~mm}$ BPM blocks connected by straight beam pipes. It is thus defined by a sequence of straight lines, which bend by half the cell angle where they join. The parameters that define the geometry are given in Table 2. The BPM blocks are 


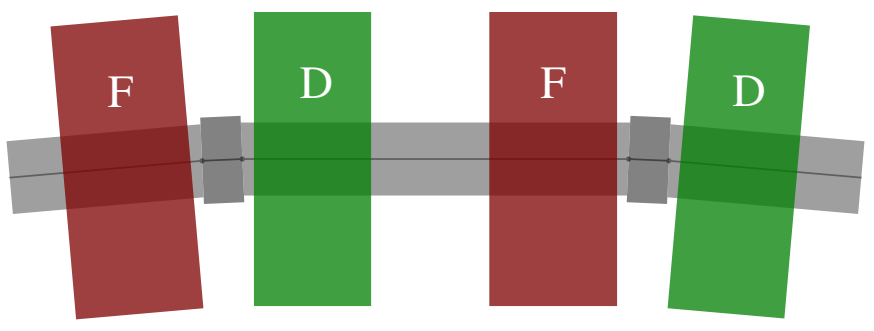

Figure 1: Illustration of FFAG arc cell geometry, showing two full cells. Lines show the reference geometry, with dots delimiting the ends of the segments. Magnet offsets are the distance of the magnet ends from the nearest dot. Segments bend by half the cell bend angle at each dot.

Table 2: Parameters for the arc cell.

\begin{tabular}{lr} 
BPM block length (mm) & 42 \\
Pipe length (mm) & 402 \\
Magnet offset from BPM block (mm) & 12 \\
Focusing quadrupole length (mm) & 133 \\
Defocusing magnet length (mm) & 122 \\
Single cell horizontal tune, $42 \mathrm{MeV}$ & 0.368 \\
Single cell vertical tune, $150 \mathrm{MeV}$ & 0.042 \\
Integrated focusing magnet strength (T) & -1.528 \\
Integrated defocusing magnet strength (T) & +1.351 \\
Integrated field on axis, defocusing (T m) & -0.03736 \\
\hline
\end{tabular}

centered in the short drift between the magnets. The precise value for the pipe length was chosen to help get the correct value of the time of flight for the entire machine.

Once the longitudinal lengths are fixed, there are three free parameters: two magnet gradients, and the dipole field in the defocusing magnet. The parameters are chosen so that the maximum horizontal closed orbit excursion at $150 \mathrm{MeV}$ and the minimum horizontal closed orbit excursion at $42 \mathrm{MeV}$, relative to the line defining the coordinate system, are of equal magnitude and opposite sign.

The remaining two degrees of freedom are used to set the tunes at the working energies. High horizontal and low vertical tunes generally reduce orbit excursions and magnet gradients. However, one must avoid the horizontal half-integer resonance at low energy and becoming linearly unstable at high energy in the vertical plane. Furthermore, we have found dynamic aperture problems near third-order resonances, in particular the $3 \nu_{x}=1$ and $\nu_{x}+2 \nu_{y}=1$ resonances. We have chosen our working point in the tune plane by considering how much gradients would need to change to reach problematic resonance lines. We quantify this change by

$$
\sqrt{\left(\frac{\Delta B_{1 F}}{B_{1 F}}\right)^{2}+\left(\frac{\Delta B_{1 D}}{B_{1 D}}\right)^{2}}
$$

where $B_{1 F}\left(B_{1 D}\right)$ refers to the gradient of the (de)focusing magnet. We find the minimum value for this quantity for values meeting the resonance condition in question, and define that to be the parametric distance. The working

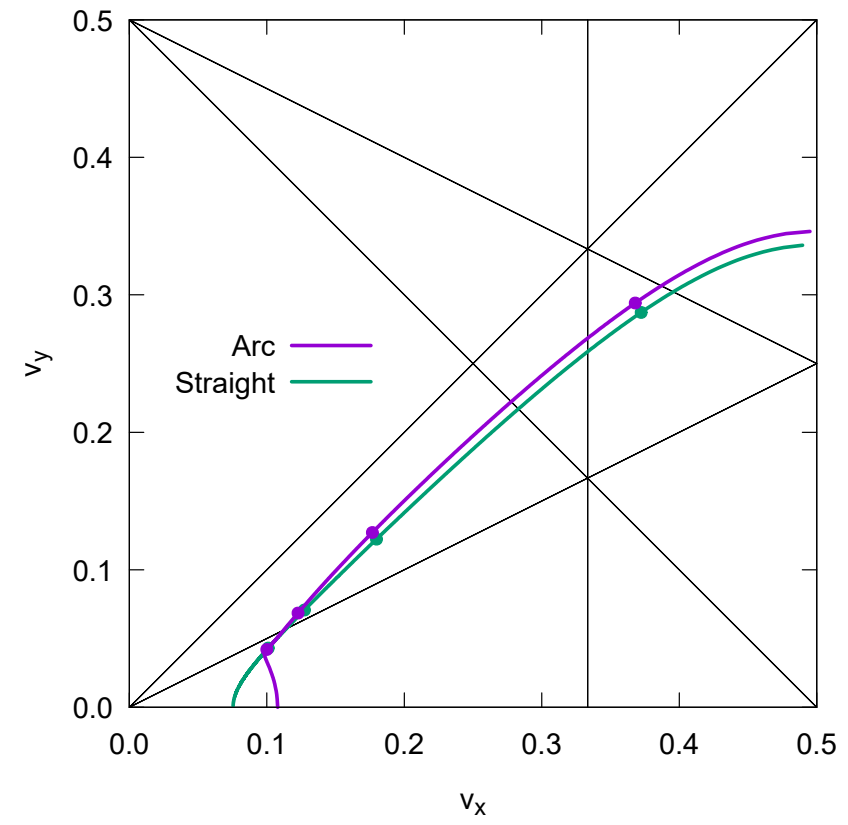

Figure 2: Tune per cell for the arc and straight cells, treated as periodic. Design energies are shown with dots. Computations are made with field maps for Halbach magnet designs.

point is chosen so that the parametric distance for of the $150 \mathrm{MeV}$ point to the $\nu_{y}=0$ line is approximately equal to the parametric distance of the $42 \mathrm{MeV}$ point to the $\nu_{x}+$ $2 \nu_{y}=0$ line, and the parametric distances of the $150 \mathrm{MeV}$ and $114 \mathrm{MeV}$ points to the $\nu_{x}-2 \nu_{y}=0$ line are about the same. The resulting working point is reasonably welldefined by the $42 \mathrm{MeV}$ horizontal and $150 \mathrm{MeV}$ vertical tunes, which are given in Table 2 . The parametric distance to the $\nu_{x}+2 \nu_{y}=0$ line is $3.0 \%$, to the $\nu_{y}=0$ line is $3.8 \%$, and to the $\nu_{x}-2 \nu_{y}=0$ line is $1.3 \%(114 \mathrm{MeV})$ and $1.2 \%$ $(150 \mathrm{MeV})$.

The computation of the paramters is performed using field maps generated by the finite element software

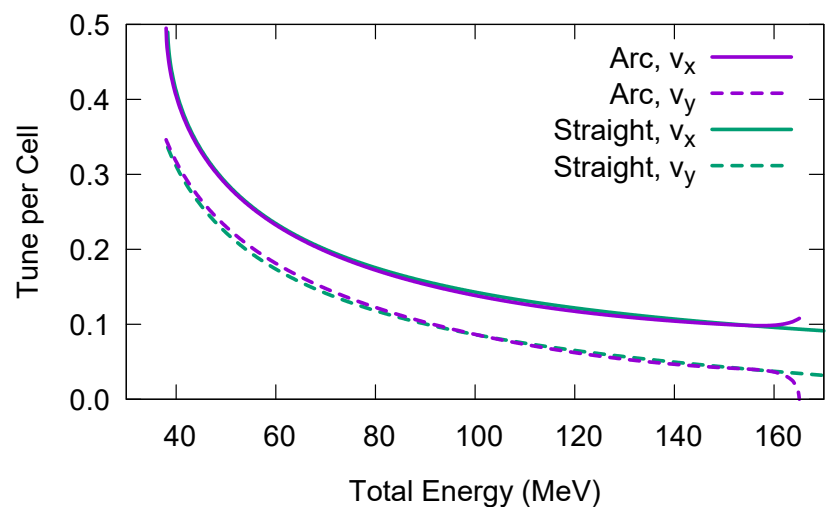

Figure 3: Tune per cell for the arc and straight cells, treated as periodic, as a function of energy. Computations are made with field maps for Halbach magnet designs. 


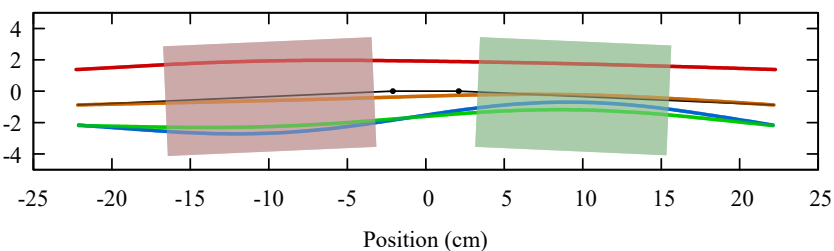

Figure 4: Periodic orbits in the arc cell. Also shown are the coordinate reference segments and the nominal magnet positions. The width of the magnets shown is equal to the pipe aperture in the midplane.

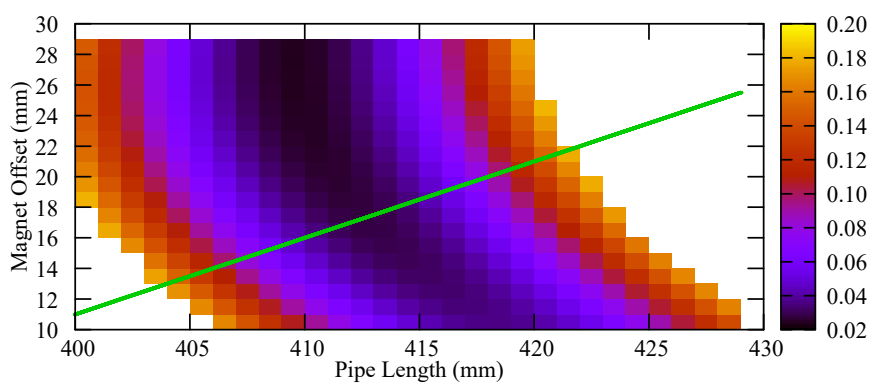

Figure 5: Quantity in Eq. (2) as a function of the pipe length (distance between the ends of the BPM blocks and offset length (distance of quadrupole ends from the ends of the BPM blocks) in the straight cell. Line drawn corresponds to a long drift length equal to that of the arc cell $(123 \mathrm{~mm})$.

OPERA. Field maps for an initial estimate for the magnet designs are created, and these field maps are scaled and shifted to achieve the desired orbit centering and tune working point. Magnet designs are then modified to have the resulting integrated gradient and central field, field maps are computed from those designs, and the results are checked (and were found to be in good agreement). Figures 2 and 3 show the tune per cell for the arc cell, and Fig. 4 shows the periodic orbits in the arc cell.

\section{Straight Cell}

The transition will adiabatically distort the lattice cell from the arc cell to a straight cell. We should thus first decide the parameters of the straight cell. To keep the transition smooth, all magnets of a given type (focusing/defocusing) will have the same integrated gradient and length. In addition, we will use the same focusing quadrupole everywhere. We will, however, use different types of defocusing magnets, differing in the integrated field on their axis. In particular, the defocuing magnet for the straight section will have zero field on its axis.

If the longitudinal lengths in the straight cell are identical to those of the arc cell, the tunes and Courant-Snyder betatron functions will be differ between the arc and the straight cells due to additional focuing occuring due to the curved paths the particles take through the arc magnets. Our goal is to make the tunes of the straight cell as close as possible to those of the arc cell. The only parameters
Table 3: Parameters for the straight cell.

\begin{tabular}{lr}
\hline BPM block length (mm) & 42 \\
Pipe length (mm) & 413 \\
Magnet offset from BPM block (mm) & 17.5 \\
Focusing quadrupole length $(\mathrm{mm})$ & 133 \\
Defocusing magnet length $(\mathrm{mm})$ & 122 \\
Straight cell count & 27 \\
\hline
\end{tabular}

available to do this are the drift lengths. The criterion used to determine the best fit is

$$
\sum_{p}\left[\sum_{i} T_{p, \operatorname{str}}\left(E_{i}\right)-T_{p, \operatorname{arc}}\left(E_{i}\right)\right]^{2}
$$

where $T_{p \text {,str }}(E)$ is the trace of the transfer matrix at energy $E$ for plane $p$ (i.e., twice the cosine of the phase advance) for the straight cell, and similarly $T_{p \text {,arc }}(E)$ for the arc cell. Fig. 5 shows this criterion plotted when varying the drift lengths. Note there is a optimum along the dark band shown in the figure. It is slightly more favorable to be toward the longer magnet offest end of that band. However, if we wish to keep the long drift length at least as long as it is in the arc, then there is a limit to how large an offset one can use. We thus choose the parameters in Table 3. The corresponding tunes are shown in Figs. 2 and 3 .

\section{Transition}

The goal of the transition is to bring the orbits in the arc at and near the design energies onto the axis in the straight. In accomplishes this by adiabatically varying the cell parameters from those in the arc to those in the straight. The adiabatic variation allows the entire energy range to end up very close to the axis in the straight. At that point, to get the correction exactly right at the design energies, the correctors can be used, and the strengths required will be very small if the transition works well.

To measure the effectiveness of the transition, we begin with the periodic orbit in the arc cell, transport it through the transition, and determine the normalized action in the straight cell when the straight cell is treated as periodic. The normalized action is

$$
J_{\text {str }}(E)=\frac{1}{2 m_{e} c}\left(\gamma_{x} p x^{2}+2 \alpha_{x} x p_{x}+\frac{\beta_{x}}{p} p_{x}^{2}\right)
$$

where $\beta_{x}, \alpha_{x}$, and $\gamma_{x}$ are the Courant-Snyder functions for the straight cell, $p$ is the total momentum for the orbit, $x$ is the horizontal position and $p_{x}$ is the horizontal momentum. The values of $J_{\text {str }}$ give an approximation to the emittance growth, and should therefore be compared to the normalized emittance of the beam, which is $1 \mu \mathrm{m}$.

Each parameter $p$ being varied has a value $p_{i}$ at cell $i$ given by

$$
p_{i}=\left[1-f_{T}\left(\frac{i}{n_{T}+1}\right)\right] p_{\text {arc }}+f_{T}\left(\frac{i}{n_{T}+1}\right) p_{\text {str }}
$$




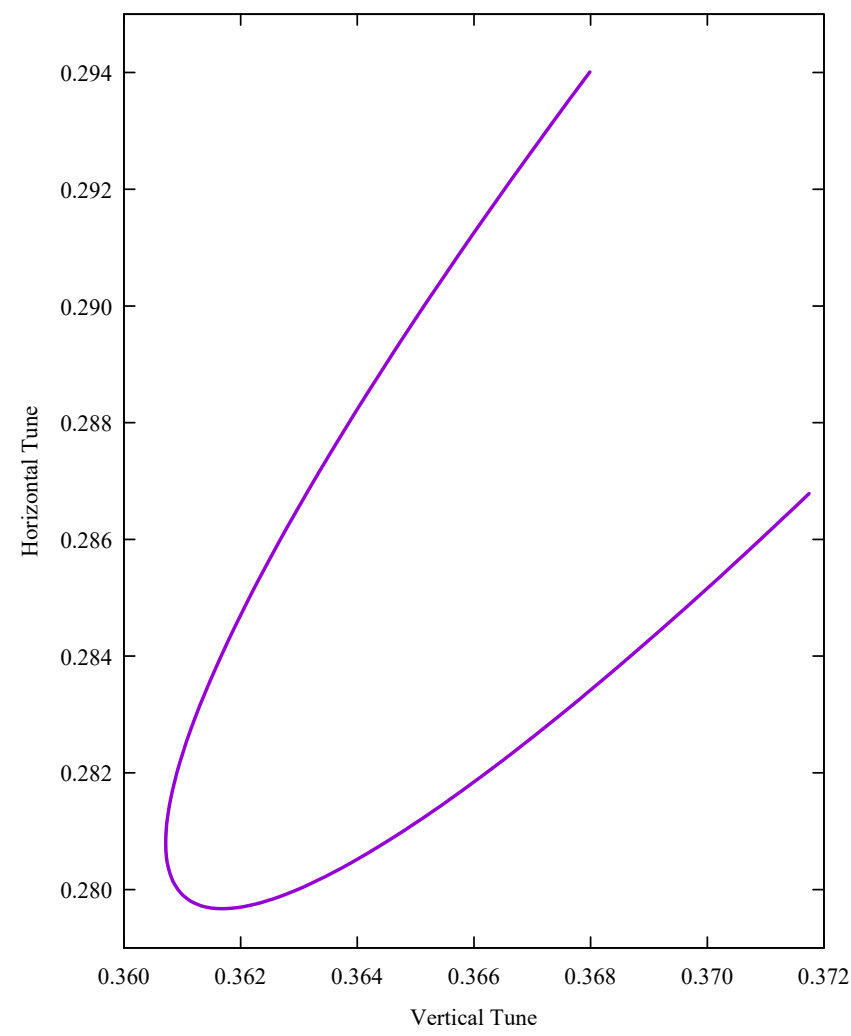

Figure 6: Tunes for a periodic cell, with angles, lengths, magnet displacements, and gradients for a hard edge model varied linearly as described in the text. Parameters vary from arc parameters to straight parameters. Computation is done using the hard-edge model described in the text.

where cell 1 is adjacent to the straight and cell $n_{T}=24$ is adjacent to the arc. The parameters varied are the lengths of the drifts, and bend angle at the BPM block, and the distance of the axis where the integrated field of the defocusing magnet is zero from the coordinate axis for the cell. The start/end of the cell is such that the distance from the end of the BPM block to the corresponding end of the cell is the same on either side of the cell.

The transition function $f_{T}$ is of the form

$$
f_{T}(x)=\frac{1}{2}+\left(x-\frac{1}{2}\right) \sum_{k=0} a_{k}\left(\begin{array}{c}
2 k \\
k
\end{array}\right) x^{k}(1-x)^{k}
$$

where we will determine the coefficients $a_{k}$ that given the best behavior. $f_{T}(1-x)=f(x)$, which is particularly useful to ensure that the average angle per cell is half the arc bend angle, which simplifies the design process by allowing the total bend angle to remain invariant as the $a_{k}$ are varied. $f_{T}(0)=0$ and $f_{T}(1)=1$ if $a_{0}=1$. If $a_{0}$ through $a_{n}$ are 1 , the function will have $n$ continuous derivatives at $x=0$ and $x=1$.

Since the phase advace is larger at lower energies, better continuity in the function will lead to smaller $J_{\text {str }}$ for lower energies. However, higher degrees of continuity at $x=0$ and $x=1$ require a steeper rise in the function around $x=1 / 2$, thus arbitrarily improving continuity at

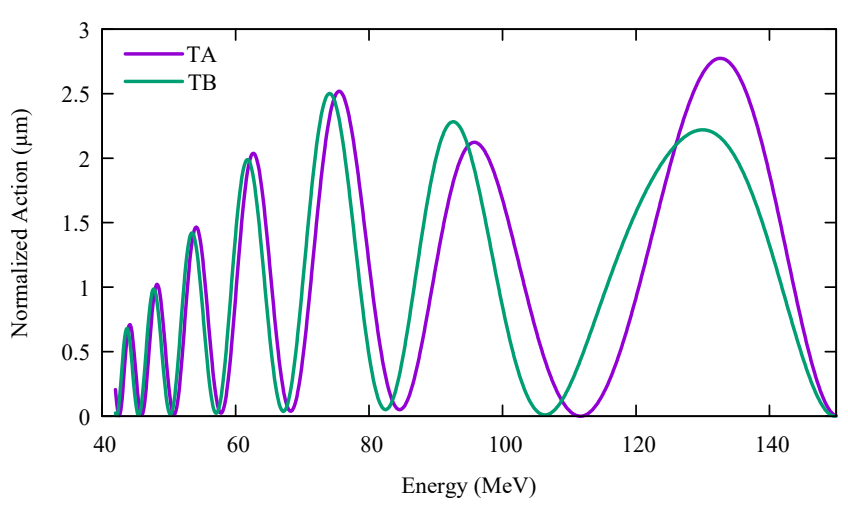

Figure 7: $J_{\text {str }}(E)$ for the transition using the taper parameterse, using a hard edge model.

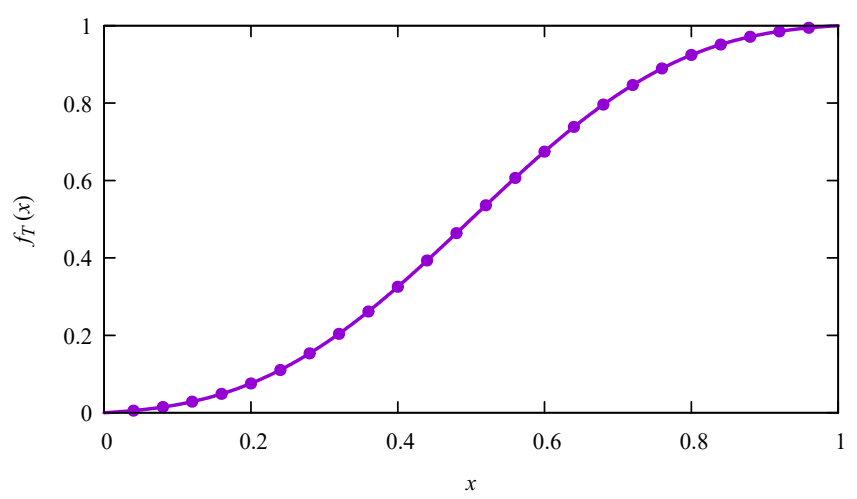

Figure 8: $f_{T}(x)$ used for the transition. Dots show the values used in for the individual transition cells.

the ends will not improve the transition performance indefinitely. Furthermore, at higher energies, where the phase advance is lower and therefore the system becomes less adiabatic, using the coefficients $a_{k}$ as adjustable parameters allows one to reduce the maximum $J_{\text {str }}$ over the entire energy range.

The linear variation in the parameters results in tunes that, rather than lying directly between the tunes for the arc and the straight, wander somewhat further from those tunes for intermediate values of $f_{T}$. However, attempting to instead keep the tunes (or the traces) on a straight line between those of the arc and the straight results in a transition with significantly worse $J_{\mathrm{str}}(E)$. It appears this is because there is a focusing term which is proportional to the square of the angle, and thus when the angle varies linearly in $f_{T}$, the focusing effect changes more rapidly at the arc end than the straight end.

In practice, I don't choose parameters that give the absolute minimum for the maximum $J_{\text {str }}$ over the energy range for a couple reasons. First, I prefer to ensure adi-

Table 4: $a_{k}$ in $f_{T}$ used for the transitions.

$a_{0}: 1.000 \quad a_{1}: 0.894 \quad a_{2}: 0.659 \quad a_{3}: 0.329$


Table 5: Magnet types used in the FFAG return line, and horizontal positions, relative to the physical magnet center, of where their integrated fields are zero.

\begin{tabular}{lr} 
BD: $27.642 \mathrm{~mm}$ & BDT2: $24.080 \mathrm{~mm}$ \\
QD: $0.000 \mathrm{~mm}$ & BDT1: $9.629 \mathrm{~mm}$ \\
\hline
\end{tabular}

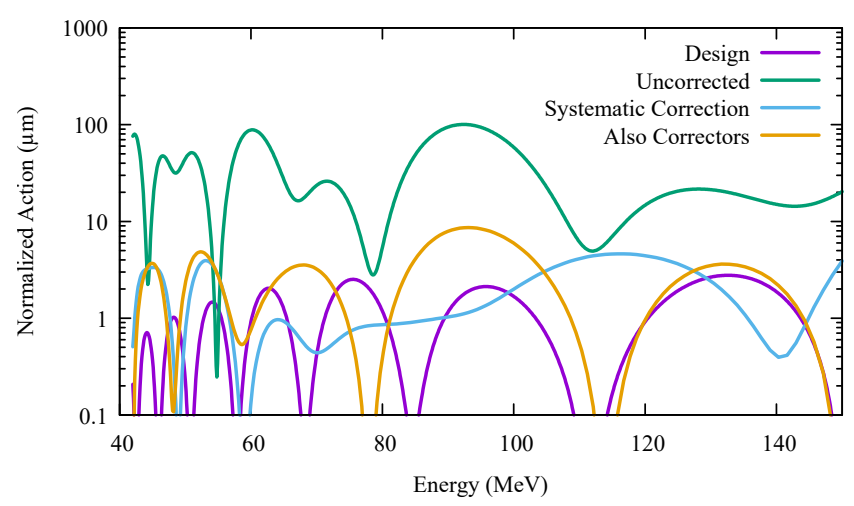

Figure 9: $J_{\mathrm{str}}(E)$ for TA. "Design" is for the hard edge model, and is the same as Fig. 7. "Uncorrected" is with field maps, when $f_{T}$ alone is used to position the magnets. "Systematic Correction" applies an additional systematic correction to each magnet type as described in the text. "Also Correctors" additional applies correctors on the QF magnets in the transition to make $J_{\text {str }}(E)$ be zero at the design energies.

abatic reduction in $J_{\text {str }}$ at lower energies rather than adjusting parameters for the absolute minimum at higher energies; this allows lower energies to in a sense take care of themselves without being dependent on the precise choice for the $a_{i}$ and fine-tuning by correctors. Second, because the doublet is not reflection symmetric in the longitudinal direction, the two transitions behave somewhat differently, and thus the optimal coefficients are somewhat different for the two transitions. However, they are close enough that it is reasonable to choose the same coefficients for both transitions, and the penalty for doing so is small.

The coefficients were optimized using a hard-edge approximation to the lattice that attempts to give a good approximation to the low and high energy tunes and orbits. The tunes and the orbit positions at the center of the long pipe are matched at the low and high energy by adjusting quadrupole and dipole fields of the hard edge model, as well as adding thin quadrupoles to the magnet ends, offset so they have the same zero field axis as the magnet they correspond to. The drift lengths are adjusted as described above, and the modeled quadrupole gradients and the offset of the zero field axis are adjusted using $f_{T}$ as well (note the gradients of the real magnets do not change). The resulting $J_{\text {str }}(E)$ is shown in Fig. 7, with the $f_{T}$ used shown in Fig. 8. The corresponding $a_{k}$ are shown in Table 4.

\subsection{Discrete Magnet Types}

The FFAG beamline uses the same focusing quadrupole throughout, but four distinct types of defocusing magnets. While all the defocusing magnets have the same integrated

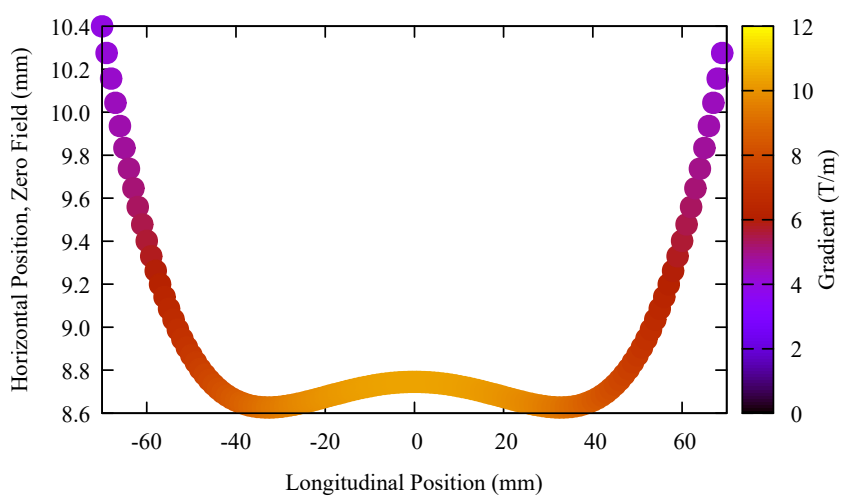

Figure 10: Points along a line where $B_{y}=0$ in the midplane of BDT1. Colors show gradient along that line.

Table 6: Additional offsets of magnets in the transition, given at the endpoints of the transition section for each defocusing magnet type. These values are for TA.

\begin{tabular}{crrrr}
\hline$f_{T}$ & $\Delta x(\mu \mathrm{m})$ & \multicolumn{2}{c}{$\Delta x(\mu \mathrm{m})$} \\
\hline 0.00 & BDT1 & +210 & QF & +220 \\
0.71 & BDT1 & +110 & QF & +100 \\
0.71 & BDT2 & +50 & QF & +150 \\
1.00 & BDT2 & -40 & QF & +40 \\
\hline
\end{tabular}

gradient, they have different integrated fields on-axis, or equivalently, a different horizontal position where the integrated field is zero. The horizontal positions where the integrated fields are zero for the different magnet types are shown in Table 5. BD is used in the arc, QD in the straight, and BDT1 and BDT2 are used in the transition.

The horizontal positions of BDT1 and BDT2 are changed depending on which cell the magnets are in, so as to have the position of the zero axis vary as described in Eq. (4). I use BDT2 when $f_{T}>0.71$ and BDT1 for $f_{T}<0.71$ so that, for each magnet the positive and negative shifts are approximately equal. The resulting $J_{\text {str }}(E)$ is shown in Fig. 9. As can be seen, the performance of the transition is significantly worse with the maps. The underlying reason is that two different magnet types, placed with their zerofield axes in the same location, do not behave precisely the same. A simple example of the problem is shown in Fig. 10. BDT1, since it is adjacent to QD in the transition, should ideally be an adequate replacement for QD, where the line where the field is zero is a straight line. But as shown in the figure, the line where the field is zero is not precisely straight. In the QD, a particle any energy will enter and exit with zero angle when starting along the axis. But in BDT1, for a particle to asymptotically start and end parallel to the magnet axis, it must start at a different horizontal position depending on its energy.

To attempt to correct for this, I add a systematic offset to BDT1 and BDT2 as well as the QF magnets in the corresponding sections. This function will be linear in $f_{T}$ 
Table 7: Offset of magnets in each transition cell.

\begin{tabular}{|c|c|c|c|}
\hline D type & $f_{T}$ & Offset, QF (mm) & Offset, D (mm) \\
\hline BDT1 & 0.0056 & 0.219 & -9.266 \\
\hline BDT1 & 0.0146 & 0.218 & -9.017 \\
\hline BDT1 & 0.0286 & 0.215 & -8.634 \\
\hline BDT1 & 0.0486 & 0.212 & -8.083 \\
\hline BDT1 & 0.0757 & 0.207 & -7.337 \\
\hline BDT1 & 0.1106 & 0.201 & -6.377 \\
\hline BDT1 & 0.1535 & 0.194 & -5.198 \\
\hline BDT1 & 0.2041 & 0.186 & -3.807 \\
\hline BDT1 & 0.2617 & 0.176 & -2.223 \\
\hline BDT1 & 0.3252 & 0.165 & -0.475 \\
\hline BDT1 & 0.3933 & 0.154 & +1.396 \\
\hline BDT1 & 0.4641 & 0.142 & +3.344 \\
\hline BDT1 & 0.5359 & 0.129 & +5.319 \\
\hline BDT1 & 0.6067 & 0.117 & +7.267 \\
\hline BDT1 & 0.6748 & 0.106 & +9.138 \\
\hline BDT2 & 0.7383 & 0.139 & -3.630 \\
\hline BDT2 & 0.7959 & 0.117 & -2.056 \\
\hline BDT2 & 0.8465 & 0.098 & -0.673 \\
\hline BDT2 & 0.8894 & 0.082 & +0.498 \\
\hline BDT2 & 0.9243 & 0.069 & +1.452 \\
\hline BDT2 & 0.9514 & 0.058 & +2.194 \\
\hline BDT2 & 0.9714 & 0.051 & +2.741 \\
\hline BDT2 & 0.9854 & 0.046 & +3.122 \\
\hline BDT2 & 0.9944 & 0.042 & +3.370 \\
\hline
\end{tabular}

for the corresponding section:

$$
\Delta x\left(f_{T}\right)=\Delta x\left(f_{0}\right) \frac{f_{1}-f_{T}}{f_{1}-f_{0}}+\Delta x\left(f_{1}\right) \frac{f_{T}-f_{0}}{f_{1}-f_{0}}
$$

For the end point in the middle, I use 0.71. The values of $\Delta x$ for the focusing and defocusing magnets at the end points for each transition section with a given defocusing magnet type ( 8 values in all) are adjusted to minimize the maximum $J_{\text {str }}(E)$ over the energy range. The resulting offsets at the endpoints are given in Table 6 , and the corresponding $J_{\text {str }}(E)$ are shown in Fig. 9.

\subsection{Applying Dipole Correctors}

Dipole correctors can be applied to get the design energies precisely correct. The goal of the taper is to bring $J_{\text {str }}(E)$ as close as possible to zero over the full energy range, to reduce the required corrector strengths required to zero $J_{\text {str }}(E)$ at the design energies, and to make the design robust against systematic errors. The correctors are then applied on top of this, and the required strengths should be small.

To compute the corrector strenghts, I used an iterative algorithm where a matrix computing the response of $x$ and $p_{x}$ at the straight for the design energies to changes in dipole corrector strengths is computed. A linear computation is made to determine approximately the changes in corrector strengths that would zero $J_{\text {str }}(E)$ at the design energies, while minimizing the sum of the squares of the changes in the corrector strengths. Starting with the corrector strenghts at zero, this algorithm is repeated until the $J_{\text {str }}(E)$ are zero at the design energies; in fact, one step of the algorithm gives a more than adequate estimate. The resulting $J_{\text {str }}(E)$ is shown in Fig. 9. The maximum required corrector strength is $16 \mu \mathrm{T} \mathrm{m}$.

\section{Appendix A. To be done}

- Update to use new magnets

- Do the transition correction for TB

- References, introduction, etc. 